\title{
Scenario Development: A Model-Driven Engineering Perspective
}

\author{
Umut Durak $^{1}$, Okan Topçu ${ }^{2}$, Robert Siegfried ${ }^{3}$ and Halit Oğuztüzün ${ }^{4}$ \\ ${ }^{1}$ Institute of Flight Systems, German Aerospace Center (DLR), Lilienthalplatz 7, Braunschweig, Germany \\ ${ }^{2}$ Department of Computer Engineering, Naval Science and Engineering Institute, Istanbul, Turkey \\ ${ }^{3}$ Aditerna GmbH, Riemerling, Germany \\ ${ }^{4}$ Department of Computer Engineering, Middle East Technical University, Ankara, Turkey \\ umut.durak@dlr.de,okantopcu@gmail.com,robert.siegfried@aditerna.de,oguztuzn@ceng.metu.edu.tr
}

Keywords: Scenario Development, Distributed Simulation, Base Object Model, Model-Driven Engineering.

\begin{abstract}
Scenario development starts with capturing scenarios from the users and leads to the design and the development of the simulation environment to execute these scenarios. This paper proposes a scenario development process adopting a Model-Driven Engineering (MDE) perspective. It takes scenario development and the use of scenarios in simulation environment development put forth in IEEE Recommended Practice for Distributed Simulation Engineering and Execution Process (DSEEP) as a starting point. It then constructs a basic vocabulary including the definitions of operational, conceptual, and executable scenarios. Following MDE principles, scenario development is viewed as a series of model transformations. Operational scenarios, mostly defined in a natural language, are first transformed into conceptual scenarios, which conform to a formal metamodel. Then conceptual scenarios can be transformed into executable scenarios specified using a specific scenario definition language. Furthermore, it is also possible to generate the constructs of simulation environment design and development using model transformations. In this regard, a conceptual scenario metamodel is proposed adopting the Base Object Model metamodel as an example. Then this metamodel is used to present the proposed process with a sample operational scenario and conceptual scenario excerpts. Samples are shown how model transformation can be employed for developing a Federation Object Model and an executable scenario file.
\end{abstract}

\section{INTRODUCTION}

Although the importance of scenarios in modelling and simulation has long been well known, there still exists a lack of common understanding and standardized practices in simulation scenario development. Scenario development starts with eliciting scenarios from the users and leads to physical scenario data representation for runtime execution and constraints to simulation environment design.

Scenario development is an extensive process beginning with the stakeholders' descriptions of the scenario and finishing with the generation of the corresponding executable specifications. The scenario development is a part of the simulation environment development process. The scenario development aims at developing a specification of a simulation run, but it is also an input for the design and development of the simulation environment itself.
Siegfried and his colleagues propose to distinguish three types of scenarios that are produced in successive stages of the scenario development process: operational scenarios, conceptual scenarios and executable scenarios (Siegfried et al., 2012) (Siegfried et al., 2013) (MSG-086, 2014).

In this paper Siegfried's definitions are used as a baseline for devising a model-driven scenario development process. This process involves establishing a scenario development pipeline. It adopts Model-Driven Engineering (MDE). MDE proposes that one shall construct a model of the system to be built and then proceed with a series of transformations to obtain an executable system (Mellor et al., 2003). Following the principles of MDE, scenario development is viewed as the transformation of operational scenarios (defined in a natural language) to conceptual scenarios (conforming to a formal metamodel) then to executable scenarios (specified using a specific scenario definition language) and simulation 
environment design (defined in a particular formalism).

After introducing the required background information, the proposed model-driven scenario development process is presented. Then the process is illustrated with a simple example.

\section{BACKGROUND}

The definition of scenario has long been a subject of discussion. Siegfried et al. (Siegfried et al., 2012) define a scenario as a specification of conditions and situations to be represented by a simulation environment for its purpose.

In IEEE 1278 (IEEE, 1993), the standard for Distributed Interactive Simulation, a scenario is defined as the description of initial conditions and timeline of significant events. The definition given in the High Level Architecture Glossary (US Department of Defense, 1996) stresses that a scenario shall identify the major entities with their capabilities, behavior and interactions over time with all related environmental conditions. The NATO Science and Technology Organization Modeling and Simulation Group 053 (MSG-053, 2010) defines a scenario as a description of the hypothetical or real area, environment, means, objectives and events during a specified time frame related to events of interest.

The operational scenarios are provided in the early stages of a simulation environment development process by the user or the sponsor. The operational scenarios can be documented in any textual or graphical form. The key elements in a scenario are the initial state, the desired end state, the course of actions to reach the prescribed end state, and the entities with their capabilities and relations.

The operational scenarios provide a coarse description of the intended situation and its dynamics, but they need to be refined and augmented with additional information pertaining to simulation. This refinement is usually done by M\&S experts and results in conceptual scenarios. Conceptual scenarios provide a detailed specification of the piece of the world to be represented in the simulation environment and should provide crucial information for everyone who is involved in the later stages of the simulation environment engineering process.

Once a simulation environment is designed and set up, the executable scenarios have to be available for all simulation systems and other member applications of the simulation environment. For this purpose, the conceptual scenarios need to be transformed into executable scenarios. An executable scenario is the specification of a specific situation providing all information necessary for the preparation, initialization, and execution of a simulation environment and for supporting scenario management activities such as scenario distribution and role casting (Topçu \& Oğuztüzün, 2010). The transformation from conceptual scenarios to executable scenarios is undertaken primarily by the operator of the member applications of the simulation environment (possibly assisted by $M \& S$ experts or subject matter experts). Ideally, the resulting executable scenarios are specified in a way that they are directly processable by the member applications (e.g. as a file containing parameters or via a web service).

\section{DEVELOPMENT PROCESS}

A standard perspective for the utilisation of scenarios in simulation development and execution is introduced in IEEE Recommended Practice for Distributed Simulation Engineering and Execution Process (DSEEP) (IEEE, 2010a). DSEEP describes a process framework for development and execution of distributed simulation environments. The DSEEP recommends scenario development activity as a part of the problem conceptual analysis. The outcome of this activity is defined as the major entities that must be represented in the simulation environment, description of their capabilities, behaviors and relationships, event timelines, the environment, and the initial and terminal conditions. The DSEEP then prescribes the utilization of scenarios: a) for the design of a simulation environment and for the design of the member applications, and b) for designing and establishing the simulation environment agreements in simulation environment development. These agreements enable the simulation applications to interoperate. From an HLA perspective, this corresponds to defining federates, a Federation Object Model (IEEE, 2010b), and Federation Agreements (Johns Hopkins University Applied Physics Laboratory, 2010).

MDE has also been employed in systems development in the simulation domain to generate elements of a simulation system or simulation environment from models via model transformations (Topçu et al., 2008) (Adak et al., 2009) (Gaševic et al., 2009) (Durak et al., 2009) (Tolk, 2002) (Cetinkaya et al., 2011). The MDE methodology is 
regarded as a natural continuation of the advances in raising abstraction level for systems development to boost productivity as well as quality (Atkinson \& Kuhne, 2003). The models are refined and transformed during the development process until an executable artifact is obtained.

Adopting this MDE definition for scenario development, the authors propose a development process in which conceptual scenarios are specified based on a metamodel and then executable scenarios for various target simulation systems are generated via model transformations employing transformation rules specified for those particular targets.

Conforming to the process model recommended by DSEEP, we promote the construction of the conceptual scenarios as models and the utilization of model transformations for designing member applications, environment agreements and executable scenarios.

\section{METAMODELING}

MDE worldview is founded on models and transformations among them. In order to describe a model, one needs a language. One way to provide a language is metamodeling. The Object Management Group (OMG) introduced a four-level metamodeling architecture, which specifies four levels: Information (M0), Model (M1), Metamodel (M2) and Metametamodel (M3), and their relations (OMG, 2011b).

M0 consists of the data to be described. M1 comprises the model that describes the data. M2 describes the structure and the semantics of the model and named as metamodel and M3 is the top level that specifies the structure and the semantics of the metamodel and named as meta-meta model.

The proposed model-driven scenario development process is structured upon this fourlayer metamodeling architecture of OMG. The process advocates constructing a Conceptual Scenario Metamodel prior to developing conceptual scenarios. The aim is to start with a metamodel to enable building a conceptual model and then to support the model transformations from the source, conceptual scenario to target simulation application design, simulation environment agreements, and executable scenario.

Figure 1 exemplifies the proposed process. It recommends to develop a completely new metamodel or to use an existing one for the shown targets depending on the simulation environment development process. The representations of target models depend on the specification languages used

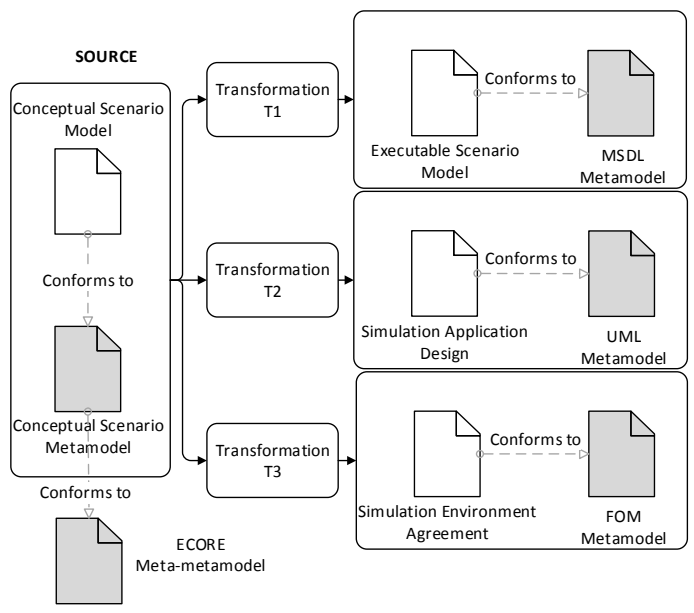

Figure 1 Model-Driven Scenario Development Process.

as depicted in the exemplified transformations in Figure 1. For instance, the design of a simulation application can be specified by using a general modeling language such as UML, or by using a more specific representation which targets a specific platform such as Federation Architecture Metamodel (FAMM) for HLA federations (Topçu et al., 2008). If the UML metamodel is the target the transformation rules will be specified from the Conceptual Scenario Metamodel to the UML metamodel. If HLA Object Models for environment agreement are used, then FOM metamodel is the target. Lastly, one can use specific scenario definition languages such as the Military Scenario Definition Language (MSDL) (SISO, 2008) as the target metamodel for executable scenarios.

In the proposed scenario development process, the conceptual scenario is subject to either model-tomodel or model-to-text transformations. To accomplish these transformations, one needs to specify the mappings between the constructs of the source metamodel and those of the target metamodel. Then a source model is transformed into a target model by executing the specified transformation (Gronback, 2009).

\section{SAMPLE IMPLEMENTATION}

In this section, the process introduced in the previous section will be elaborated using a sample implementation. In this implementation, we will first introduce metamodeling over a conceptual scenario metamodel that has been constructed adopting Base Object Model (BOM) (SISO, 2006) metamodel.

Eclipse Modeling Framework (EMF) is 
employed to realize the four-level metamodeling architecture. EMF is defined as a framework for describing models and then generating other constructs, such as other models, code or text from them (Steinberg et al., 2008).

Next, a sample conceptual scenario vignette will be introduced using the conceptual scenario metamodel. Finally, transformation definitions will be discussed over the sample mappings for generating FOM and the executable scenarios from the conceptual scenario.

\subsection{Conceptual Scenario Metamodel}

Base Object Model (BOM) introduces the interplay, the sequence of events between simulation elements, as well as the reusable pattern, and provides a standard to capture the interactions (SISO, 2006). Siegfried and his colleagues presented BOMs as a method for capturing conceptual scenarios (Siegfried et al., 2013). Following this approach, we adopt the BOM metamodel specified in the standard to construct a conceptual scenario metamodel.

Ecore is provided as the meta-metamodel used to describe metamodels in EMF (Steinberg et al., 2008). To define a metamodel, one makes use of four Ecore classes, namely, EClass, EAttribute, EReference and EDataType. EClass is defined as the modelled class with attributes and references. EAttribute is the modelled attribute with a name and a type. EReference is specified as an association between classes. EDataType is the type of an attribute (Steinberg et al., 2008).

At the top level (Figure 2) ConceptualScenario, defined as an EClass, has associations that are defined by EReference constructs: entities, stateMachines, interplays, events and identification. These relate ConceptualScenario to other EClasses Conceptual Entity, StateMachine, PatternOf Interplay, Event and ScenarioIdentification, respectively.

ScenarioIdentification has attributes that are defined by POCEmail, POCTelephone and so on as EAttributes. As an example the Purpose attribute is defined as a string (EString) data type (EDataType).

A conceptual scenario is defined with one or more state machines. A state machine is defined by a number of states. Each state has an exit condition and a next state. Exit conditions are associated with exit actions that are pattern actions. For example, in a flight simulation, the aircraft conceptual entity may have six states: taxi, takeoff, climb, cruise, descend and landing. The exit condition for taxi can be defined as the takeoff clearance given by the

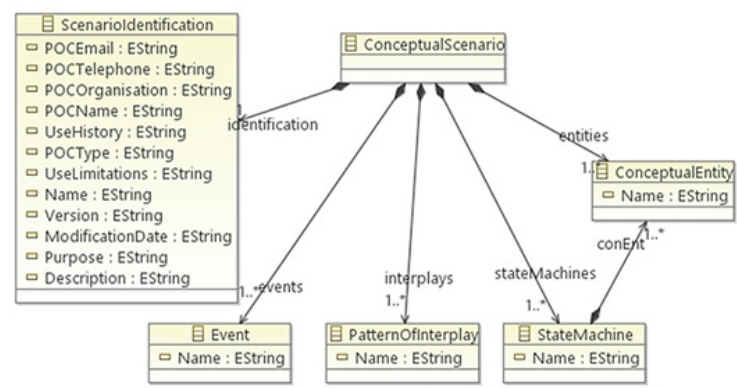

Figure 2: Conceptual Scenario Metamodel Top Level Diagram.

control tower. Then takeoff can be specified as the next state.

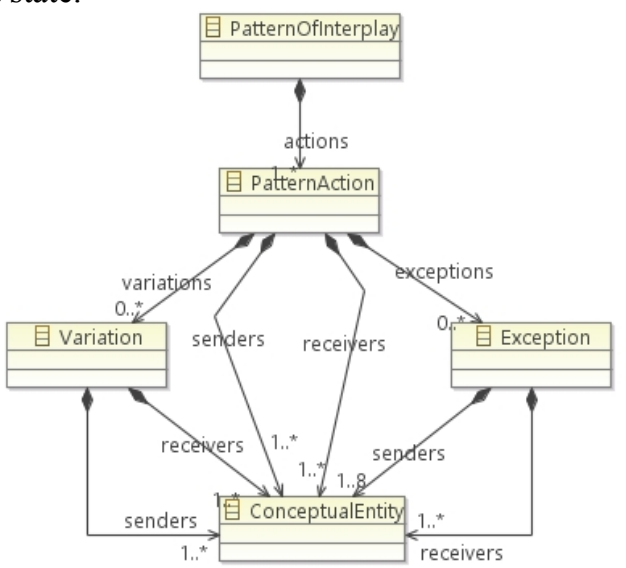

Figure 3: Pattern of Interplay in Conceptual Scenarios.

Patterns of interplay are defined as building blocks in the BOM specification (SISO, 2006). They capture the pattern actions as well as their exceptions and variations. As shown in Figure 3, actions are initiated by sender conceptual entities and receivers are the intended recipients. Exceptions are defined as the actions that cause the remaining sequence to fail. Variations, however, are defined as alternative ways of an action that do not affect the completion or success. Considering again the case of an aircraft, the pattern of interplay for the departure is likely to include the aircraft beginning to roll from its parking position in the direction of the assigned runway. Depending of the parking position (in front of the Terminal or further away on the Apron) an initial push-back might be required or not, which can be introduced as a variation of this action. The sender for the taxiing can be specified as the pilot and the receiver as the aircraft. The second action can then be defined as getting the clearance from the control tower. The sender in this case is the control tower and receiver is the pilot and the event is the takeoff clearance. And the pattern of interplay can 
continue with an action of applying power to the aircraft's engine.

In BOM metamodel, conceptual entities take part in patterns of interplay as senders and receivers and each entity is associated with a state machine. Entities possess characteristics and the BOM metamodel is enhanced by adding values to these characteristics to define scenario parameters. For example, various characteristics can be specified for an aircraft entity in a flight simulation scenario, some of which are initial position, fuel weight or gross weight. The values of these characteristics then determine the scenario parameters.

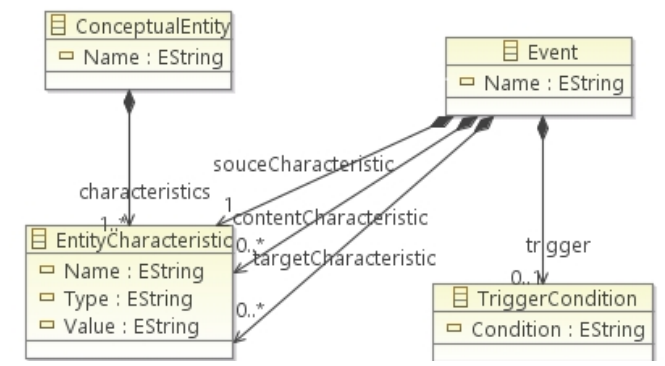

Figure 4: Conceptual Entities and Events in Conceptual Scenarios.

Events (Figure 4) are used to capture the messages and triggers. Triggers present undirected events when a change in the characteristic of an entity creates a response from other entities. The condition of change is captured in a trigger condition. Messages are directed events from one entity to another that are uniquely identified by the source and target characteristic. The content of a message is given in content characteristics. As an example, takeoff clearance is a message from tower (which is identified by its airport id) to an aircraft (which is identified by its call sign). The content of the message is the takeoff clearance characteristic of the aircraft. When the takeoff characteristic of an aircraft is true, then it is a trigger event. Then the pilot entity can start an action for gears up.

\subsection{Model-Driven Conceptual Scenario Development}

This section is based on an operational scenario for the departure activity of an aircraft. Below is an extract from the operational scenario.

"Aircraft D-ATRA stands in front of its hangar at $D L R$ in Braunschweig. Pilots ask the tower for taxi clearance. The tower provides taxi instructions towards RWY 08. Pilots then start taxiing according to instructions. Then tower provides information about the departure like weather, VRB05KT, R08/2800FT, overcast sky. Then pilots ask for a departure clearance and tower grants the departure." (DFS Deutsche Flugsicherung GmbH, 2013) (Ahmad \& Sexana, 2008).

This is an example operational scenario one can obtain from the users or sponsors of a flight simulator. It is obvious that not all data that is required to run this scenario is available in this text. An M\&S expert needs to augment the missing information and to develop a conceptual scenario.

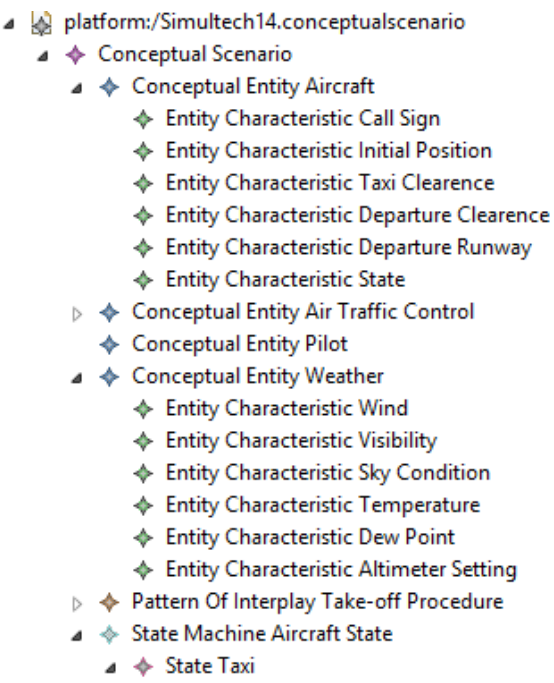

Figure 5: Conceptual Scenario Editor Tree Viewer.

EMF.Edit (Steinberg et al., 2008) is employed to build conceptual scenario editor via automatic code generation to display and edit the instances of the developed metamodels. This editor provides a tree viewer and properties sheet for each conceptual scenario element. Figure 5 presents the tree viewer for the sample conceptual scenario. The tree presents some of the conceptual entities from the operational scenario such as aircraft, pilot, and weather. The main pattern of interplay is defined as takeoff procedure. Aircraft state is captured as a statechart. When developing the conceptual scenario, missing weather characteristics in operational scenario such as temperature and dew point are also added to the model.

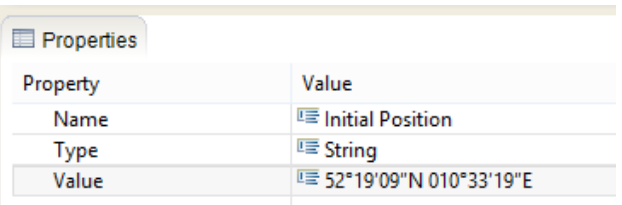

Figure 6: Conceptual Scenario Editor Properties Viewer. 
SIMULTECH 2014 - 4th International Conference on Simulation and Modeling Methodologies, Technologies and Applications

The Properties viewer (Figure 6) enables a user to specify the attributes of the model elements. As an example, the attributes of initial position entity characteristics are its name (initial position), type (string), and value $\left(52^{\circ} 19^{\prime} 09^{\prime \prime} \mathrm{N} 010^{\circ} 33^{\prime} 19^{\prime \prime} \mathrm{E}\right)$. Thus an $M \& S$ expert can specify the implicit reference to the initial location of the aircraft in the operational scenario explicitly.

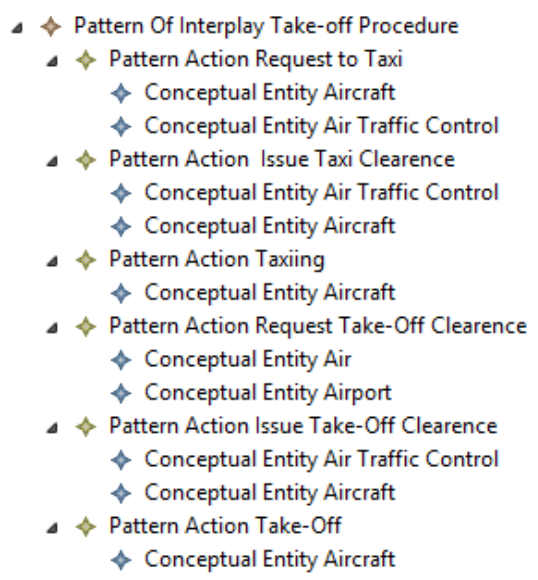

Figure 7: A Sample Pattern of Interplay.

The takeoff procedure is presented in Figure 7 as a sample pattern of interplay. There are six consecutive actions, starting from "aircraft requests to taxi from Air Traffic Control" till its takeoff. The sender and receiver entities are all captured. Even though exceptions and variations can be specified, this sample does not exhibit any.

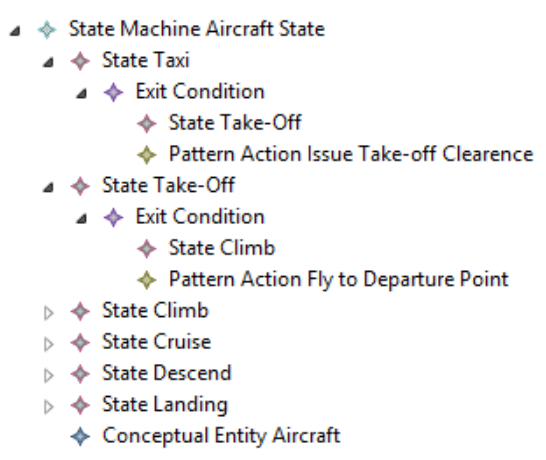

Figure 8: A Sample State Machine.

Figure 8 introduces a sample aircraft state machine. Aircraft states include taxi, takeoff, climb, cruise, descend and landing. The next state after taxi is takeoff and the exit action of the taxi state is the issue of takeoff clearance. The next state after takeoff is climb and takeoff ends with the action flying to departure point.

\subsection{Model Transformations}

Model transformations are the enabling tools of MDE for development. Throughout the engineering process, models are the main artifacts, and transformations enable the reflection of the information captured in one model to another one as well as enriching the source model with specialized information. In the model-driven scenario development process model transformations are proposed for transforming the information that is captured in a conceptual model to simulation environment design, simulation environment agreements and executable scenarios. To define transformations from a source metamodel to a target metamodel, a model transformation language is required. Atlas Transformation Language (ATL) (Jouault et al., 2006), Graph Rewriting and Transformation (GReAT) (Agrawal, 2003) and Query / View / Transformation (QVT) (OMG, 2011a) are some of the commonly used languages. Rather than addressing any specific model transformation language, users are recommended to pick any model transformation language that fits their specific requirements such as the development environment requirements or target model requirements.

Here, the sample transformation specification, or mapping, was developed using QVT utilizing Eclipse Model-to-Model Transformation (MMT) project (The Eclipse Foundation, 2014). It supports a QVT Operational, a partial implementation of the QVT specification (Barendrecht, 2010).

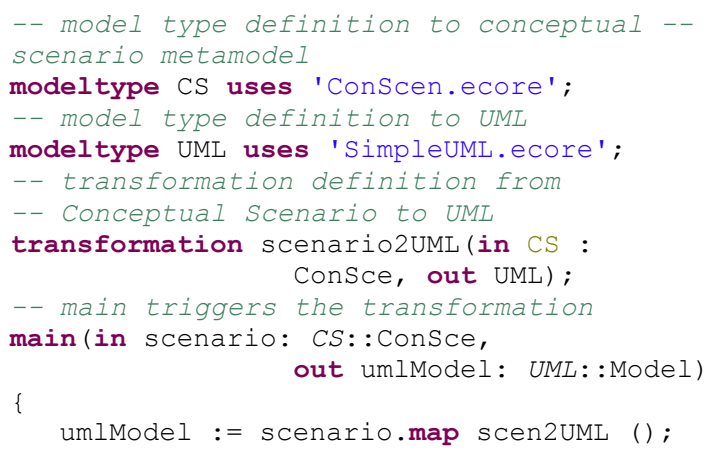

As presented above, QVT defines transformations using a certain structure, which consists of model type definitions, transformation declarations, and a main function. Metamodels are referred by using model type definitions. Transformation declarations specify the input and the output metamodels. The main function starts the transformation process by calling the first transformation. 
Mappings specify which object from an instance of a source metamodel will be transformed to which specific object in the instance of the target metamodel. The declarations identify the source class name and the target class name. One can also specify constraints to mappings using Object Constraint Language (OCL) (OMG, 2006). In the body of a mapping, the variables and the parameters are initialized in the init section, mappings are specified in the population section and postprocessing can be done in the end section.

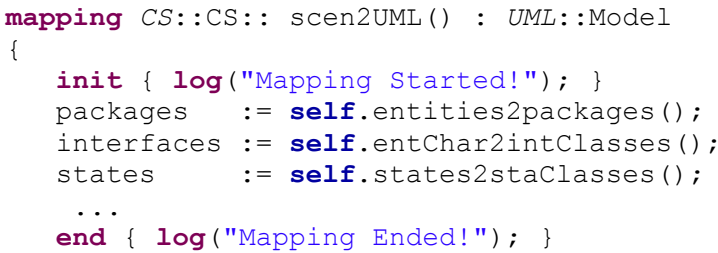

Above is a representative excerpt from the top level mapping that is called in the main function. Conceptual entities in the conceptual scenario metamodel are mapped to packages in the UML model calling a new mapping function self.entities2packages(). Similarly, the entity characteristics and the states in the conceptual scenario metamodel are mapped to the interface classes and to the state classes, respectively, in the UML model.

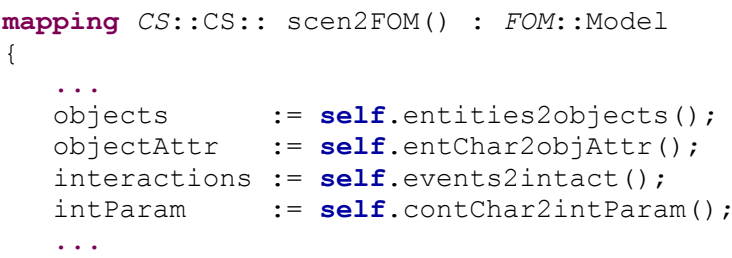

Likewise, a sample portion is provided for the conceptual scenario to Federation Object Model transformation. In this case entities can be mapped to HLA objects and entity characteristics to object attributes. Events in the conceptual scenario metamodel can be mapped to HLA interactions in a FOM and content characteristics to interaction parameters.

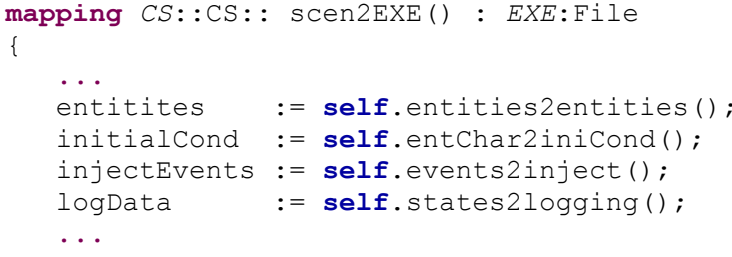

For a transformation to create an executable scenario from a conceptual scenario, mappings need to be specified as exemplified above. Entities in the conceptual scenario must be mapped to the entities of the executable scenario.

\section{CONCLUSIONS}

This paper introduced a model-driven scenario development process which is based on the explicit specification of conceptual scenarios using a metamodel. The proposed development process recommends the use of model transformations to generate the executable scenarios. Practitioners of this process shall develop their metamodels for the source (i.e. conceptual scenario) and target (i.e. executable scenario, design of simulation applications, or simulation environment agreements).

The proposed process is illustrated with a simplified case study. In this respect, BOM presents a prospect in specifying the conceptual scenarios as the source metamodel. Target metamodels on the other hand are more or less application-specific. The examples introduced in this paper made use of Eclipse-based technologies for modeling and transformation,although there exist alternatives to Eclipse for each of these steps.

In order to elaborate on the proposed modeldriven scenario development process, an effort to develop the corresponding workflows will be worthwhile.

\section{REFERENCES}

Adak, M., Topçu, O. \& Oğuztüzün, H., 2009. Modelbased Code Generation for HLA Federates. Software: Practice and Experience, 40(2), pp.149-75.

Agrawal, A., 2003. GReAT: A Metamodel Based Model Transformation Language. Institute for Software Integrated Systems (ISIS), Vanderbilt University.

Ahmad, S. \& Sexana, V., 2008. Design of Formal Air Trafic Control System throug UML. Ubiquitous Computing and Communication Journal, 3(6), pp.1120.

Atkinson, C. \& Kuhne, T., 2003. Model-driven development: A metamodeling foundation. IEEE Software, 20(5), pp.36-41.

Barendrecht, P.J., 2010. Modeling transformations using QVT Operational Mappings. Research project report. Eindhoven: Eindhoven University of Technology Department of Mechanical Engineering Systems Engineering Group.

Cetinkaya, D., Verbraeck, A. \& Seck, M.D., 2011. MDD4MS: A Model Driven Development Framework 
SIMULTECH 2014 - 4th International Conference on Simulation and Modeling Methodologies, Technologies and Applications

for Modeling and Simulation. In Summer Simulation Conference. The Hague, Netherlands, 2011.

DFS Deutsche Flugsicherung $\mathrm{GmbH}, 2013$. Aerodome Chart -ICAO Braunschweig-Wolfsbug. Langen: DFS Deutsche Flugsicherung GmbH.

Durak, U., Oguztuzun, H. \& Ider, K., 2009. Ontology Based Domain Engineering for Trajectory Simulation Reuse. International Journal of Software Engineering and Knowledge Engineering, 9(8), pp.1109-29.

Gaševic, D., Djuric, D. \& Devedžic, V., 2009. Model Driven Engineering. In Model Driven Engineering and Ontology Development. Berlin: Springer.

Gronback, R.C., 2009. Eclipse Modeling Project: A Domain-Specific Language. Upper Saddle River, NJ: Addison-Wesley.

IEEE, 1993. IEEE 1278 Protocols for Distributed Interactive Simulation Applications-Entity Information and Interaction. Standard. New York, NY: IEEE.

IEEE, 2010a. IEEE Recommended Practice for Distributed Simulation Engineering and Execution Process (DSEEP). New York, NY: IEEE.

IEEE, 2010b. IEEE Standard for Modeling and Simulation High Level Architecture (HLA)- Object Model Template (OMT) Specification. Standard. New York, NY: IEEE.

Johns Hopkins University Applied Physics Laboratory, 2010. Live-Virtual-Constructive Architecture Roadmap Implementation, Common CapabilitiesFederation Agreements Template Users' Guide. Technical Report. Laurel, MD: Johns Hopkins University.

Jouault, F. et al., 2006. ATL: a QVT-like Transformation Language. In OOPSLA '06. New York, 2006. ACM.

Mellor, S.J., Clark, A.N. \& Futagami, T., 2003. Guest editors' introduction: Model-driven development. IEEE Software, 20(5), pp.14-18.

MSG-053, 2010. Rapid Scenario Generation for Simulation Applications. RTO Technical Report. Brussels: NATO.

MSG-086, 2014. Guideline on Scenario Development for (Distributed) Simulation Environments. STO Technical Report. Brussels: NATO.

OMG, 2006. Object Constraint Language, Version 2.0. Standard. Needham, MA: OMC Object Management Group.

OMG, 2011a. Meta Object Facility (MOF) 2.0 Query/View/Transformation Specification. Standard. Needham, MA: OMG.

OMG, 2011b. Object Management Group, Meta object facility,MOF specification version 2.4.1. Standard. Needham, MA: OMG.

Siegfried, R. et al., 2012. Scenarios in military (distributed) simulation environments. In Spring Simulation Interoperability Workshop (S-SIW). Orlando, 2012. SISO.

Siegfried, R. et al., 2013. Specification and documentation of conceptual scenarios using Base Object Models (BOMs). In Spring Simulation Interoperability Workshop. San Diago, 2013. SISO.

SISO, 2006. SISO-STD-003-2006 Base Object Model
(BOM) Template Specification. Orlando, FL : Simulation Interoperability Standards Organization (SISO).

SISO, 2008. Standard for Military Scenario Definition Language (MSDL). Standard. Orlando, FL : SISO.

Steinberg, D., Budinsky, F., Merks, E. \& Paternostro, M., 2008. EMF: Eclipse Modeling Framework. 2nd ed. Upper Saddle River, NJ: Pearson Education.

The Eclipse Foundation, 2014. Model-to-Model Transformation (MMT). [Online] Available at: https://projects.eclipse.org/projects/modeling.mmt [Accessed 10 February 2014].

Tolk, A., 2002. Avoiding another Green Elephant - A Proposal for the Next Generation HLA based on the Model Driven Architecture. In Fall Simulation Interoperability Workshop. Orlando, FL, 2002.

Topçu, O., Adak, M. \& Oğuztüzün, H., 2008. A Metamodel for Federation Architectures. Transactions on Modeling and Computer Simulation (TOMACS), 18(3), pp.10:1-10:29.

Topçu, O. \& Oğuztüzün, H., 2010. Scenario Management Practices in HLA-based Distributed Simulation. Journal of Naval Science and Engineering, 6(2), pp.133.

US Department of Defense, 1996. High Level Architecture Glossary. US DoD: Washington DC, VA. 